\title{
Some reflections about the success and impact of the computer algebra system DERIVE with a 10-year time perspective
}

\author{
Eugenio Roanes-Lozano · Jose Luis \\ Galán-García • Carmen Solano-Macías
}

Received: date / Accepted: date

\begin{abstract}
The computer algebra system DERIVE had a very important impact in teaching mathematics with technology, mainly in the 1990's. The authors analyze the possible reasons for its success and impact and give personal conclusions based on the facts collected. More than 10 years after it was discontinued it is still used for teaching and several scientific papers (most devoted to educational issues) still refer to it. A summary of the history, journals and conferences together with a brief bibliographic analysis are included.
\end{abstract}

Keywords Computer algebra systems · DERIVE - educational software · symbolic computation · technology in mathematics education

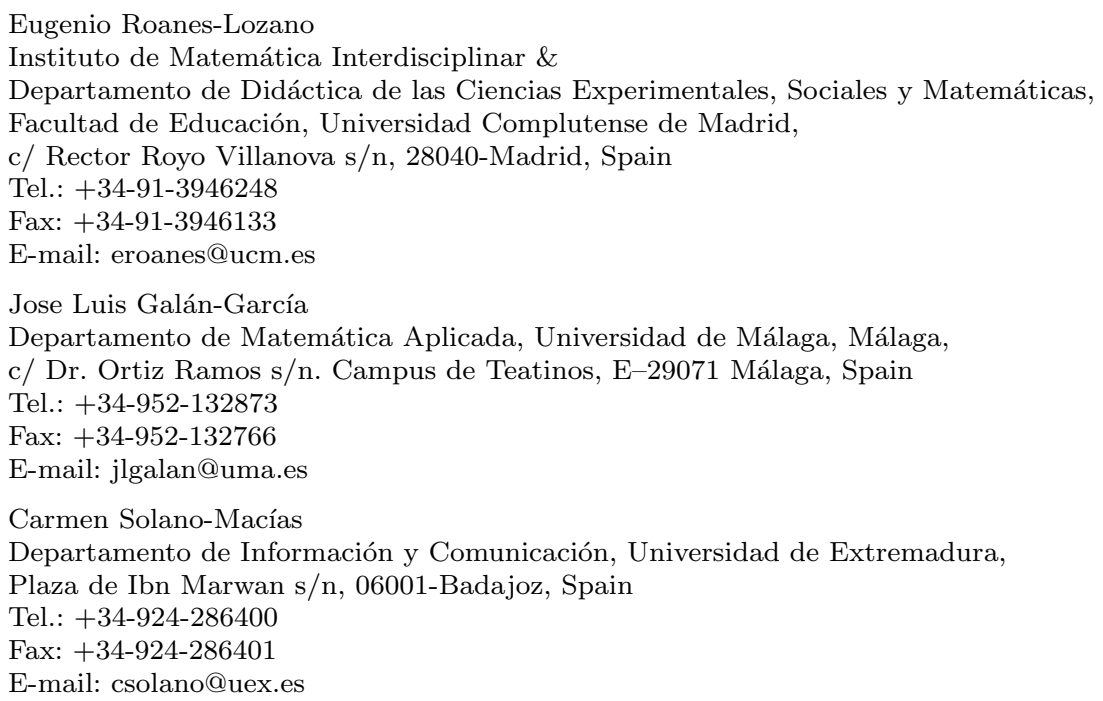




\section{Introduction}

Undoubtedly, software evolves at a very high speed. Moreover, meanwhile in the past the launch of new versions only followed drastic changes in the software, nowadays many pieces of software systematically present a new version every year (that supersedes the previous one).

In markets different from the software one, like the automobile sector, not being able to anticipate and guess the new tendencies can produce a decline in sales. In the software market everything occurs very fast and some sales leaders have followed a quick decline or "extinction" for the same reasons. For instance, regarding word processors, the "de facto standard" WordStar (launched in 1978) [56,57] was substituted in this role by WordPerfect [55], itself replaced by $M S$-Word.

Another curious fact is the relatively low proportion of the market taken by free compatible software (sometimes excellent and comparable to the commercial ones). That is the case, for instance, of the word processors OpenOffice [52] and FreeOffice [47].

Regarding the computer algebra systems (CAS) $[22,42,16]$, the decline of the two pioneers of the 60's, Macsyma [32,5,31], now alive as Maxima, and REDUCE [18-20,29,33], would deserve an in-depth study. Even if they later evolved in parallel with the nowadays leaders Mathematica and Maple, they lost their leadership. Maxima [51] and REDUCE [54] can now be obtained free of charge.

Sometimes, the lack of a critical mass of users can make a powerful piece of software to decline and finally fall into abandonment. That is what probably happened with the revolutionary CAS AXIOM [45], where the algebraic structure where the computations have to be performed is fixed by the user [23].

DERIVE was released in 1988 by Software House and is now owned (although discontinued) by Texas Instruments.

We shall focus on the following questions: What has happened with $D E$ RIVE after being abandoned by Texas Instruments, when it was very popular (if not at the peak of its popularity)? Has it followed a steep decline? Which are the reasons for the behaviour observed in the DERIVE community?

Although there are very many articles, books and proceedings about $D E$ RIVE and its applications, for instance $[1,4,6-15,21,25,39,40]$, none, as far we are aware, is devoted to analyze the success and slow decline of this piece of software.

As said above, although the possibilities of the CAS DERIVE and its educational applications have been analyzed in many articles, it hasn't been studied from the point of view of the analysis of its success and remanence. Therefore, we believe that the topic addressed can be considered a novelty. 


\section{A brief overview of DERIVE's history}

At the end of the '70s Albert D. Rich, a logician and software developer, had designed and implemented a dialect of LISP, muLISPr9 [34], that run on small computers: the $m u(\mu)$ stands for micro.

Together with Prof. David Stoutemyer they founded in 1979 The Software House in Honolulu (Hawaii), with the goal of creating small yet powerful CAS. Their first product was the command-line interface CAS muMATH'79 [37], written in muLISP. Its last version was muMATH83. It was rally small and its hardware requirements were very limited.

Surprisingly, as early as 1983 Klaus Aspetsberger and Gerhard Funk run in an Austrian high school an educational experiment with the CAS ( $m u M A T H)$ [6] that is probably the world's first of its kind.

Later Stoutemyer and Rich developed what became a most successful CAS: DERIVE (firstly released in 1988). Again designed for $M S$-DOS text terminals, it had comfortable wisely organized menus (see Figure 1) $[35,36,24]$. It didn't even require the computer to have a hard disk, only a $5 \frac{1}{4}{ }^{\prime \prime}$ disk unit (it didn't need to be installed).

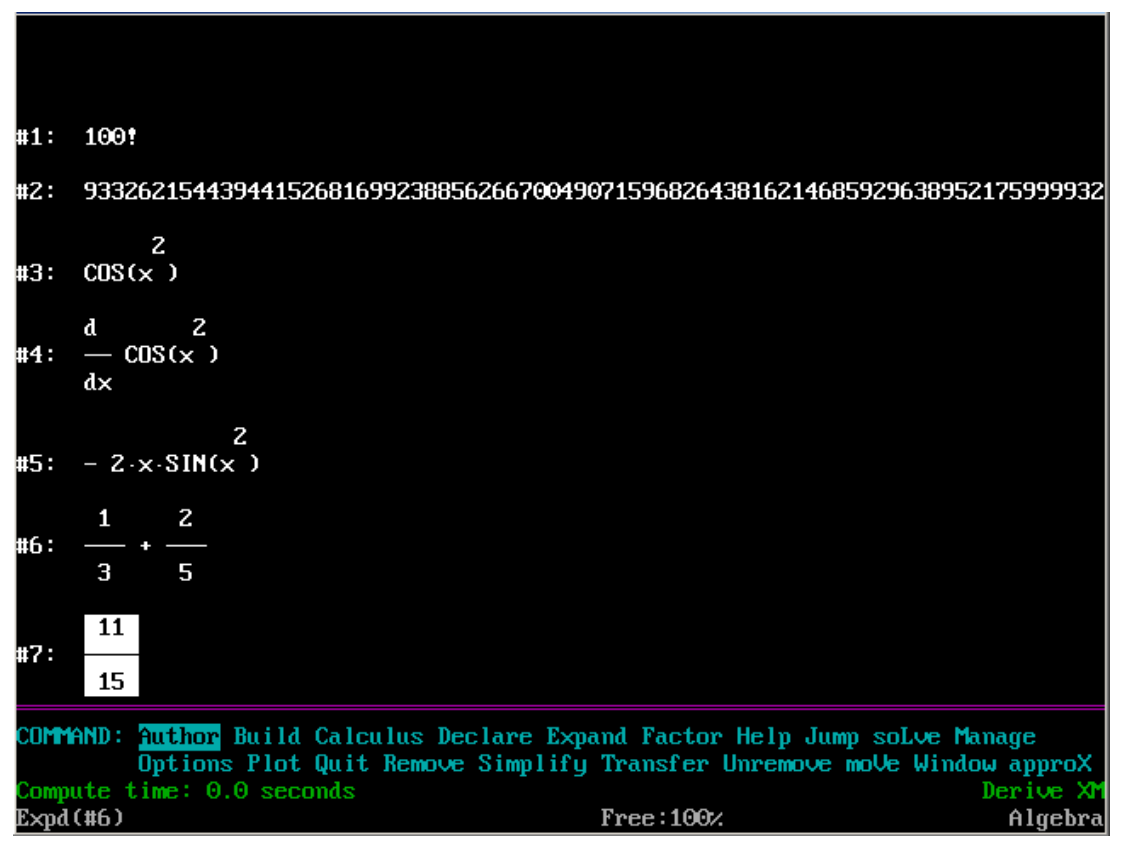

Fig. 1 Derive 3.13 user interface showing some calculations using exact arithmetic (involving big numbers and fractions) as well as some symbolic computations.

It also had 2D and 3D graphic capabilities and could plot functions in a different or spitted screen (Figure 2). 


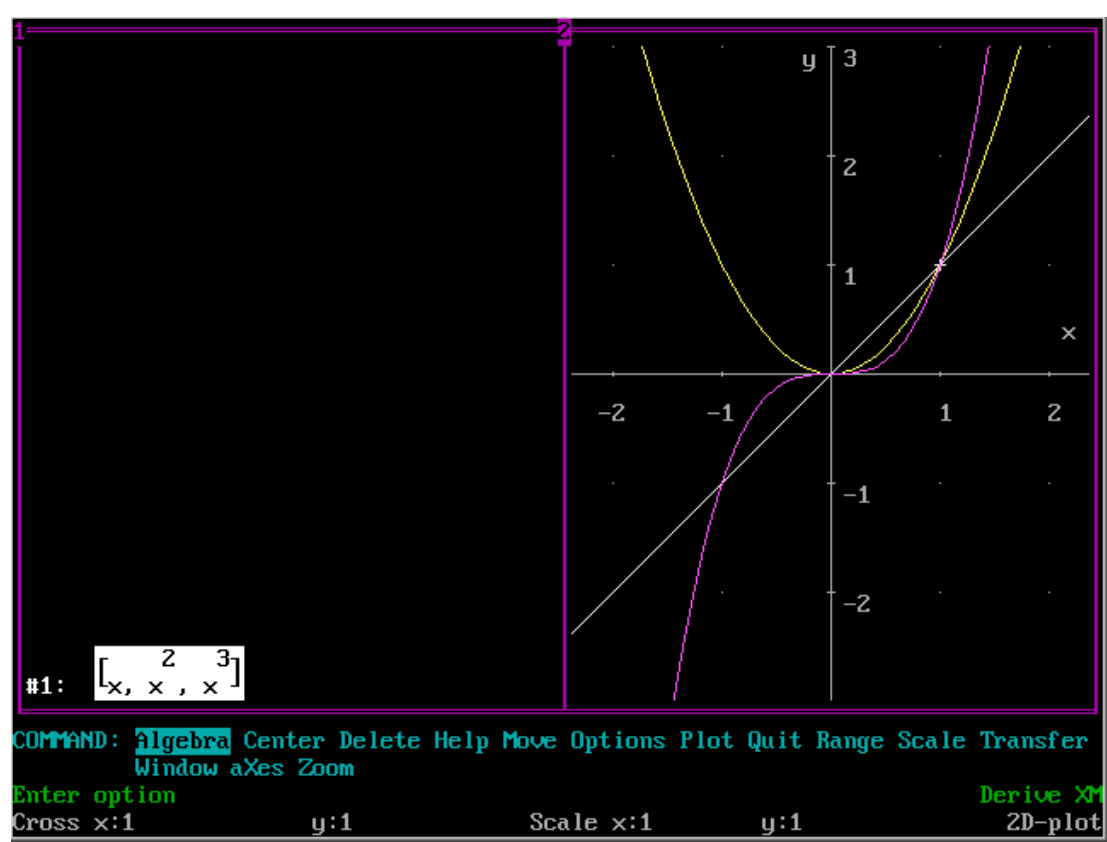

Fig. 2 Derive 3.13 user interface showing the screen split vertically (Algebra Window / Plot Window) and the plots of the functions in a given list.

DERIVE was immediately a great success, and began to be used at universities and high-schools all around the world. A remarkable case is the Austrian educational authorities, that convinced of the strong interest of using CAS in mathematics education, created the specialized research center ACDCA (Austrian Center for Didactics of Computer Algebra) [44] and acquired a Secondary Education country-wide DERIVE license.

Many educational experiments with DERIVE followed around the world. A well known book about teaching mathematics with DERIVE is [25].

As novelty, DERIVE 4 added a Windows version to the MS-DOS one.

In 1999 The Software House was acquired by Texas Instruments. The windows interface was much improved in the next version, DERIVE 5 (released in 2000): Theresa Shelby joined the team and was mainly involved with the development of the graphic interface [27].

DERIVE's final version was Derive 6.1 for $M S$-Windows (Figure 3) [28]. Although distributed in a CD, it still occupied little more than $2 M B$.

DERIVE was discontinued in 2007 in favor of TI-Nspire CAS, a product designed and developed by Texas Instruments [41,53].

A software derived from DERIVE was used in some Texas Instruments' pocket calculators like the TI-92 and TI-89 [38].

A detailed history of the software and the people involved in its design, development and spread can be found in [26]. 


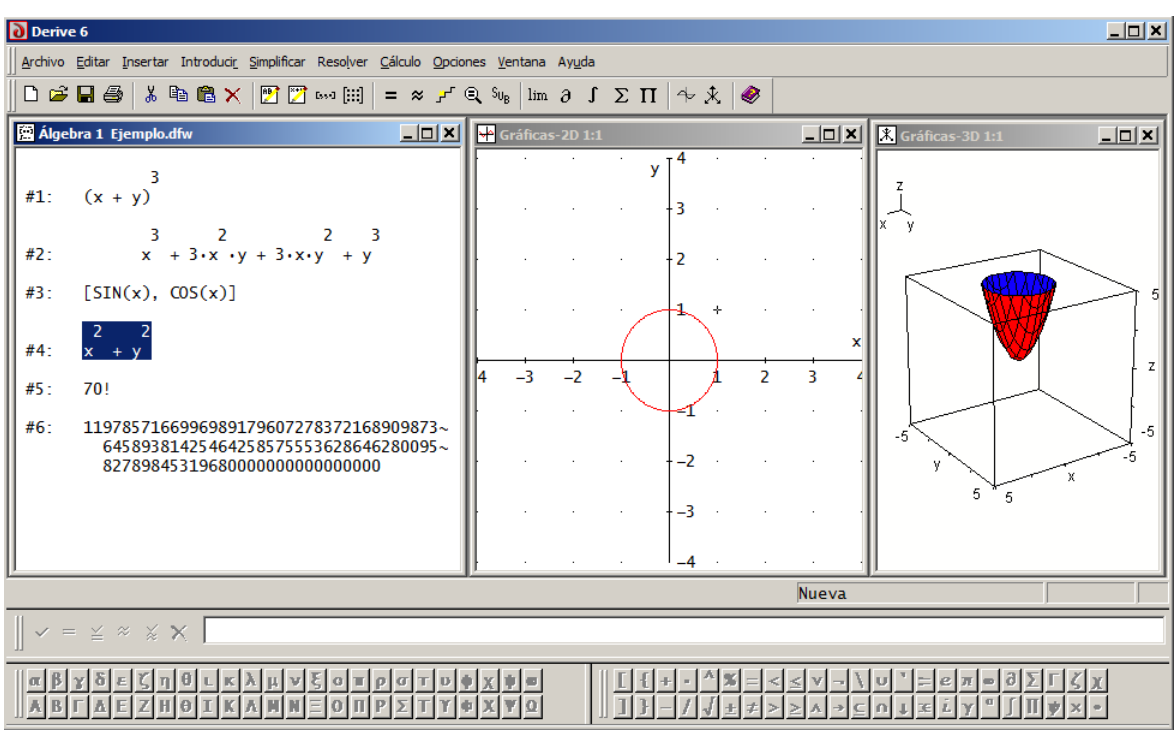

Fig. 3 Derive 6.1 user interface showing some computations, a parametric 2D plot and an explicit 3D plot.

\section{An overview of DERIVE's academic popularity}

As said before, DERIVE was abandoned by Texas Instruments in 2007, when it was very popular (if not at the peak of its popularity). Has it followed a steep or a slow decline?

\subsection{DERIVE-related conferences}

There were, of course, talks related to DERIVE presented at generic computer algebra conferences, such as ISSAC [49] and $A C A$ [43], but there were conferences much more closely related to DERIVE.

In 1994 a series of biannual DERIVE conferences began:

- The $1^{\text {st }}$ International Derive Conference took place at Plymouth, UK.

- The following conference was renamed $2^{\text {nd }}$ International Derive and TI-92 Conference, and took place in Bonn, Germany.

- The name of the following ones was again changed to International Derive 85 TI-89/92 Conference and took place in Gettysburg, USA (1998); Liverpool, UK (2000); Vienna (2002) and Montreal (2004).

- It was again renamed in 2006 as International Derive and TI-CAS Conference, and the following ones took place at Dresden, Germany (2006) and Buffelspoort, South Africa (2008).

- In 2010 the conference took place at Málaga, Spain. The order of the CAS in the title was exchanged to: International TI-Nspire $\&$ Derive Conference. 
- In 2012 the word DERIVE was dropped from the title and the scope of the conference was widened to Conference for CAS in Education 85 Research (Former International TI-Nspire \& Derive Conference). They took place at Tartu, Estonia (201) Krems, Austria (2014) and Mexico City (2016).

Meanwhile the $A C D C A$ organizes annual conferences (denoted Summer Academy) that deal primarily with didactic issues connected with the use of technology. They began one year before the DERIVE conferences, and took place simultaneously in most occasions. The list of ACDCA conference is: Krems 1992; Krems 1993; Honolulu 1995; Saeroe Hus, Kungsbacka 1996; Gösing 1998 and Portoroz 2000 [44].

From 2004 onwards, the ACDCA Summer Accademy meetings were organized together with the DERIVE-related international conference and the whole meetings were denoted Technology and its Integration in Mathematics Education (TIME 2004, TIME 2006,..., TIME2016).

A 56 pages long (!) document including the authors and titles of the lectures presented at the DERIVE and ACDCA conferences (updated to 2010) can be found at [50].

In 2018 there has been no TIME conference.

Summarizing, related conferences have been around almost 9 years after DERIVE was discontinued.

The first two authors were plenary speakers at TIME conferences. The second author was the chairmen of TIME'2010 conference and co-chair of the DERIVE strand of TIME for several years.

\subsection{DERIVE-related bulletins and journals}

Regarding journals and bulletins strongly related to DERIVE, we could mention two.

Josef Böhm, an active Austrian secondary school teacher founded The International DERIVE User Group (DUG) in 1991 and has uninterruptedly published four issues a year since then. Initially the bulletin was printed and sent to subscribers, but now only the (free) electronic version is available at [46]. It contains technical and didactic papers about DERIVE and the symbolic calculators from Texas Instruments.

The name of the $D U G$ bulletin $(D N L)$ has slightly changed along time:

- The Derive Newsletter. The Bulletin of the Derive User Group.

- The Bulletin of the Derive User Group + TI92 (1996).

- The Bulletin of the Derive User Group + CAS-TI (2003).

The $D U G$ nowadays has more than 500 members.

Not much later (1994) a journal devoted to DERIVE was founded at Plymouth University, although it has widened its aims and scope twice:

- The International Derive Journal.

- The International Journal of Computer Algebra in Mathematics Education (IJCAME) (1997) 
- The International Journal of Technology in Mathematics Education (IJTME) [48].

The second author is a member of the editorial board of this journal and has edited special issues of IJTME with selected papers from TIME'2010, TIME'2012, TIME'2014 and TIME'2016 conferences.

As a summary, the bulletin is devoted to DERIVE and alive (with a long queue of articles to be included in future issues) and the journal is fully operative (although its scope has been widened), and is indexed in Scopus and Emerging Sources Citation Index.

\section{A brief bibliographic data analysis about the evolution of DERIVE}

We are interested in knowing the evolution of the bibliographic citations related to DERIVE. We have used the scientific databases Google Scholar, Scopus and MathEduc, since we think these are the best three databases related with the scope of a CAS mainly used in mathematics education.

It is difficult to make precise searches since the word "derive" is polysemic and very frequent in English (even in the mathematic environment, as expressions such as "you can derive that" are very common). For instance, a search in Google Scholar returns thousands of useless results. Even a search for the string of characters ' Derive 3' ' returns hundreds of spurious results.

We have been testing and comparing different filters in the databases mentioned above as we have tried the data obtained to be as accurate as possible.

Although not all searches are exhaustive (in the sense that not all publications will be detected), our goal is to check the evolution of the number of documents, so exhaustiveness is not so important.

Not all databases offer exactly the same filters, sometimes the filters used are slightly different. They have also been changed in one occasion due to the very small number of results obtained in one of the searches. Again, as the goal is not to compare the results among the different databases but the evolution of the works related to DERIVE, this is not a drawback. Therefore we'll have to manage to precise the searches.

We have grouped the results in five-year periods. As we are in 2018 it has to be taken into account that the last period is shorter.

\subsection{A first bibliographic data analysis about the evolution of DERIVE}

Let us try some first searches.

- As David Stoutemyer and Al Rich appear as authors of the user manual, and Stoutemyer is an uncommon family name, a search in Google Scholar for '"DERIVE') AND ''Stoutemyer', AND '"Rich', AND ' algebra' will produce only some spurious results (although it is clear that many positive 
Table 1 Results of different bibliographic searches about DERIVE.

\begin{tabular}{llll}
\hline & $\begin{array}{l}\text { "DERIVE" AND } \\
\text { "Stoutemyer" } \\
\text { AND "Rich" } \\
\text { AND "algebra" }\end{array}$ & $\begin{array}{l}\text { DERIVE AND } \\
\text { Stoutemyer } \\
\text { AND Rich } \\
\text { AND algebra }\end{array}$ & $\begin{array}{l}\text { Rich AND } \\
\text { Stoutemeyer }\end{array}$ \\
& Search anywhere & $\begin{array}{l}\text { Search all fields } \\
\text { (Scopus) }\end{array}$ & $\begin{array}{l}\text { Search in references } \\
\text { (Scopus) }\end{array}$ \\
& (Google Scholar) & (Scour & 1 \\
& 6 & 0 & 4 \\
$1975-1979$ & 3 & 1 & 3 \\
$1980-1984$ & 10 & 1 & 12 \\
$1985-1989$ & 34 & 9 & 9 \\
$1990-1994$ & 24 & 4 & 0 \\
$1995-1999$ & 17 & 0 & 0 \\
$2000-2004$ & 19 & 0 & 0 \\
$2005-2009$ & 24 & 1 & 30 \\
$2010-2014$ & 12 & 1 & 0 \\
$2015-$ Aug 2018 & $149(153)$ & 17 & \\
\hline Total: & & & \\
\hline
\end{tabular}

results will be ignored). Such a search produces the results shown in the first column of Table 1 (149 results include a date out of 153).

The peak occurs in 1990 - 1994, but there is a surprising revival in $2010-$ 2014. The cites haven't really sensibly decreased up to 2014. Some spurious results have been detected that correspond to publications only by David Stoutemyer where the word "derive" (not referring to DERIVE) and the adjective "rich" and the substantive "algebra" can be found. It would be better to perform more refined searches.

- The same search (in "all fields") in Scopus (DERIVE AND Stoutemyer AND Rich AND algebra) returns 17 results (see the second column of Table 1).

- Only two results are obtained in MathEduc ("anywhere").

- But if we look in Scopus for articles including Rich AND Stoutemyer in their references we obtain 30 results (see the third column of Table 1). This search is more precise, as it will most probably lead to DERIVE user's manuals. They are listed in the third column of Table 1.

In the data of the two last columns of Table 1, the peak occurs in 1990 - 1994 and the shape of the corresponding polygonals resemble a Gauss bell curve (see Figure 4).

4.2 A brief bibliographic data analysis about the evolution of DERIVE in mathematics education

A search that we expected not to produce spurious results is ' CAS DERIVE' ' AND ' 'mathematics education'.

- We thought that imposing the word "CAS" just before "DERIVE" would imply to refer to the computer algebra system DERIVE. Nevertheless, as 


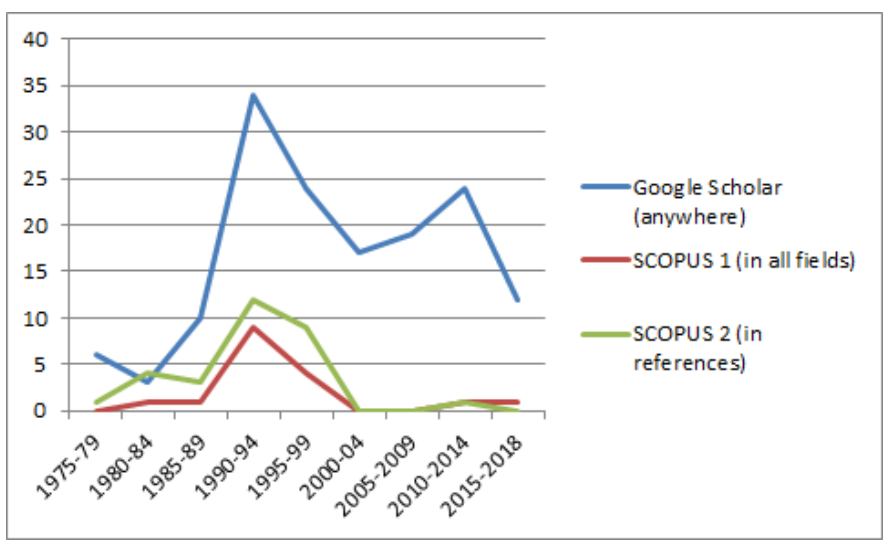

Fig. 4 Searches in Google Scholar ("anywhere") for: DERIVE $\wedge$ Stoutemyer $\wedge$ Rich $\wedge$ Algebra; search in Scopus (1) (in "all fields") and in Scopus (2) (in references) for: Rich $\wedge$ Stoutemyer. The graph corresponds to Table 1.

Google Scholar searches in the whole text (if available), some spurious results in French including "dans le cas derive" turned out to be found. Therefore we refined the search to "CAS DERIVE', AND ' mathematics education', - 'dans le cas' '. This way 104 results (99 of them dated) were obtained (see the first column of Table 2 ). The peak occurs later than other searches (2005-2009), what is reasonable, as the educational applications can be developed only later than the required software is available and known. The shape of the corresponding polygonal resembles an asymmetric Gauss bell curve, with a lower slope in its RHS (Figure 5).

- If we make the corresponding search in Scopus (search for an exact phrase): \{CAS DERIVE\} AND \{mathematics education\}, no result is obtained. Therefore, we have decided to perform a not so strict search (search for a loose or approximate phrase) with the same words: 'CAS DERIVE', AND ' 'mathematics education', and only 2 results have been obtained, surprisingly in $2015-2018$ (see the second column of Table 2). We have manually checked that they are correct.

- The results found in MathEduc including the string 'CAS DERIVE', AND ' 'mathematics education', are only 3, and all are dated after year 2000 (see the third column of Table 2).

In this case the peaks take place surprisingly recently $(2005-2009$ and $2015-2018$ ), what implies that the system is not dead or at least is taken into account when thinking about CAS and mathematics education (Figure 5). 
Table 2 Results of different bibliographic searches about DERIVE and mathematics education. Note that the search made with Scopus is a so called search for a loose or approximate phrase.

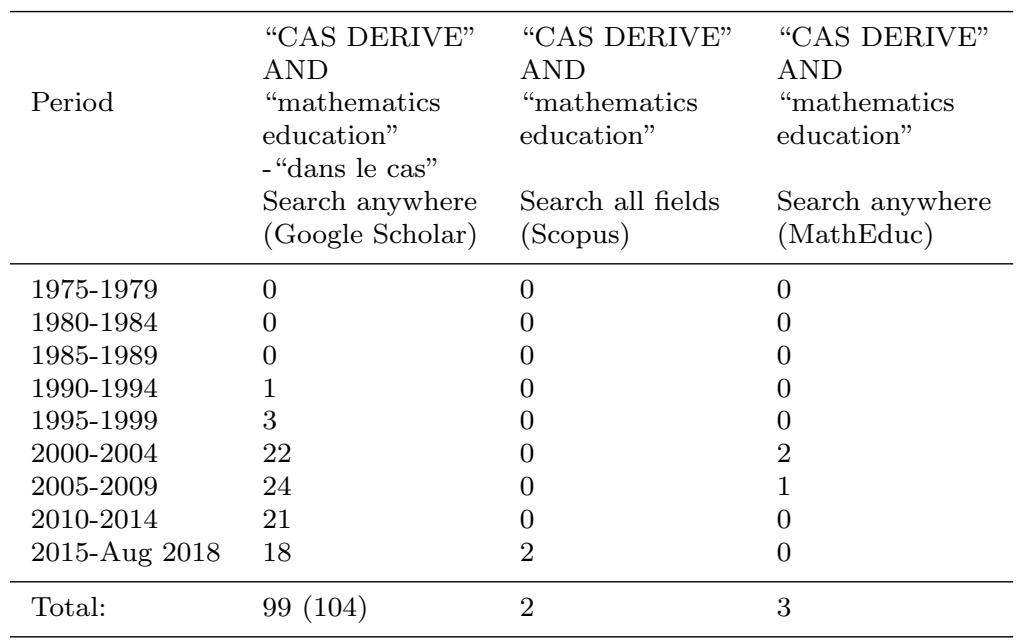

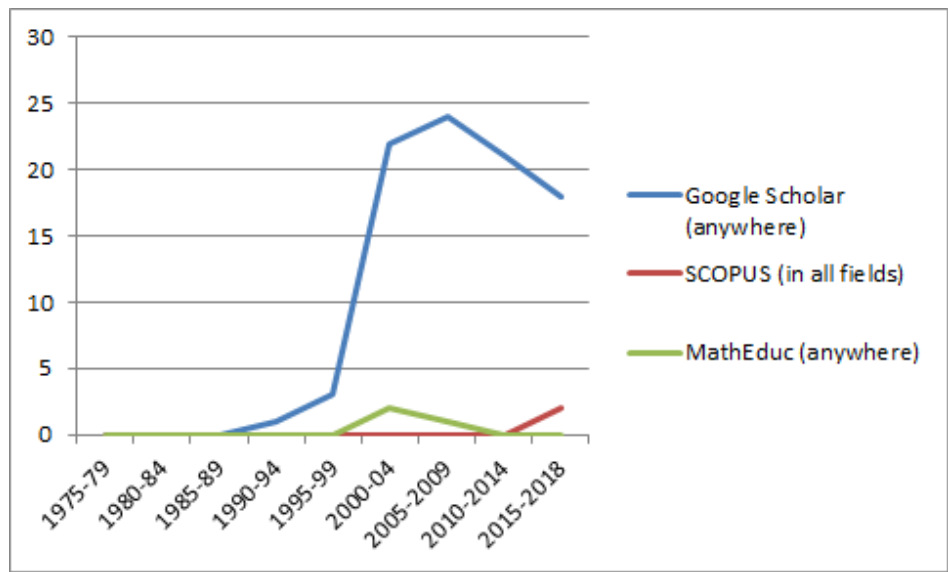

Fig. 5 Searches in Google Scholar ("anywhere") for: CAS DERIVE^ mathematics education $\wedge \neg$ dans le cas; and in Scopus (in "all fields") and MathEdu (anyhwere) for $C A S$ DERIVE $\wedge$ mathematics education. The graph corresponds to Table 2.

4.3 A brief bibliographic data analysis about the evolution of DERIVE for Windows

Unlike what happens with the polysemic string ' DERIVE' ', the string ' DERIVE for Windows', allows to make a simple and absolutely precise search in any database (looking for exact matches). 
Table 3 Some exact bibliographic searches for "DERIVE for Windows".

\begin{tabular}{llll}
\hline & $\begin{array}{l}\text { "DERIVE for } \\
\text { Windows" } \\
\text { Search anywhere } \\
\text { (Google Scholar) }\end{array}$ & $\begin{array}{l}\text { \{DERIVE for } \\
\text { Windows } \\
\text { Search all fields } \\
\text { (Scopus) }\end{array}$ & $\begin{array}{l}\text { "DERIVE for } \\
\text { Windows" } \\
\text { Search anywhere } \\
\text { (MathEduc) }\end{array}$ \\
\hline $1975-1979$ & 0 & 0 & 0 \\
$1980-1984$ & 0 & 0 & 0 \\
$1985-1989$ & 0 & 0 & 0 \\
$1990-1994$ & 1 & 0 & 0 \\
$1995-1999$ & 32 & 2 & 9 \\
$2000-2004$ & 55 & 3 & 3 \\
$2005-2009$ & 22 & 0 & 1 \\
$2010-2014$ & 19 & 2 & 0 \\
$2015-$ Aug 2018 & 6 & 0 & 0 \\
\hline Total: & $135(146)$ & 7 & 13 \\
\hline
\end{tabular}

- This new search in Google Scholar obtains 146 results, 135 of them dated (see the first column of Table 3), and its peak occurs later than other searches $(2000-2004)$, what is reasonable, as DERIVE for Windows was developed later than DERIVE. The shape of the corresponding polygonal resembles an asymmetric Gauss bell curve, with a lower slope in its RHS (see Figure 6).

- If we make the same search in Scopus instead (search for the exact phrase \{DERIVE for Windows $\}$ ) it just produces 7 results (see the second column of Table 3).

- The articles indexed in MathEduc including the string ' 'Derive for Windows', are 13 (see the third column of Table 3 ). The peak takes place in $1995-$ 1999.

In this case the three searches are almost identical (only that Google Scholar looks within the whole text (if available), what neither Scopus nor MathEduc do, should be taken into account).

The quick decay in this case (compared to others in this study) could possibly be justified because no author will nowadays specify that he refers to an application "for windows" (it is understood by default), as command line operating systems are almost abandoned.

4.4 A brief bibliographic data analysis about the evolution of the applications of DERIVE

Now we would like to filter the results corresponding to applications of $D E$ $R I V E$. We believe that a good filter is to consider publications containing in its title the strings ' 'with DERIVE') or ' 'using DERIVE' '. Although many works will be left out, the results obtained will be accurate.

- The advanced search performed in Google Scholar for " with DERIVE', OR ' 'using DERIVE' ' in the titles of the publications returns 136 results, 


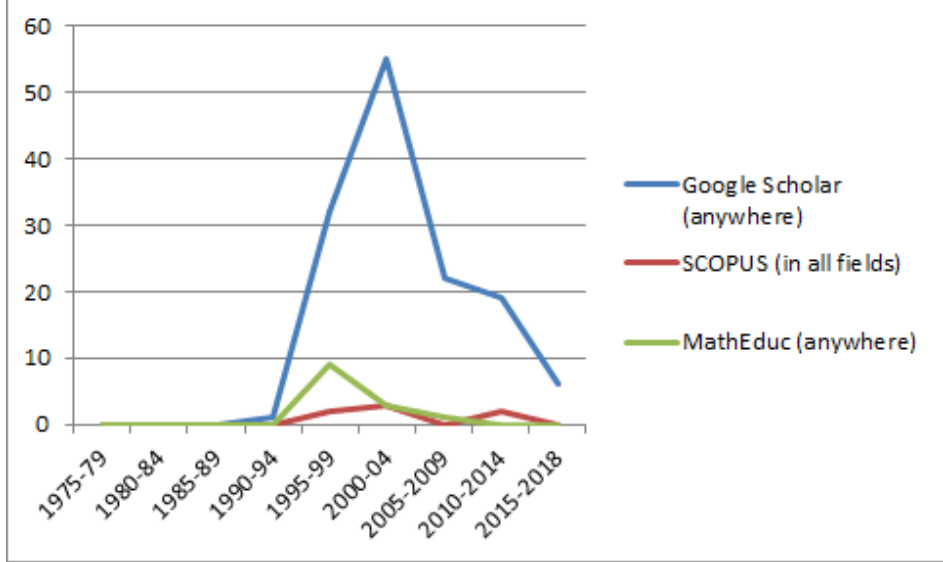

Fig. 6 Search for the string: DERIVE for Windows in Google Scholar ("anywhere"); Scopus (in "all fields") and in MathEdu (anyhwere). The graph corresponds to Table 3.

130 of them dated (see the first column of Table 4). They have its peak at $1995-1999$. The shape of the corresponding polygonal is a Gauss bell curve, truncated in its LHS (see Figure 7).

- Unfortunately, the same (exact) search in titles of publications in Scopus ( $\{$ with DERIVE $\}$ OR \{using DERIVE $\}$ ) just produces four results (see the second column of Table 4$)$.

- Nevertheless, there are many more results in Scopus if we widen the search space. For instance, if we extend the same search from "titles" to "all fields", 71 results are obtained. They can be found classified in the third column of Table 4, that is far more meaningful from the statistical point than the data in the second column. Note that such a widened search is not performed in Google Scholar because it produces more than 1000 results, some spurious, that would have to be manually filtered. The shape of the corresponding polygonal (see Figure 7) is surprisingly flat (with oscillations), showing no decline at all (and a peak at 2010-2014!). We believe that this alternative search is acceptable here, since what we are considering is the evolution of the number of publications.

- Finally, the search in MathEduc for ' with DERIVE' ' OR ' 'using DERIVE' ' in the titles of the publications returns 152 results, and shows a peak in 1995 - 1999 (see the fourth column of Table 4). The shape of the corresponding polygonal is a again a Gauss bell curve, truncated in its LHS (see Figure 7). The same results as when this search takes place in Google Scholar.

The polygonals representing the searches in titles are almost identical for Google Scholar and Scopus. Note that if we look at the polygonal corresponding to the search in Scopus for "all fields" instead of "in titles", the peak appears much later. That is because the first, second and fourth columns of Table 4 refer to works using themselves DERIVE, meanwhile publications 
Table 4 Similar bibliographic searches in Google Scholar, Scopus and MathEduc for applications of DERIVE.

\begin{tabular}{|c|c|c|c|c|}
\hline Period & $\begin{array}{l}\text { "with DERIVE" } \\
\text { OR } \\
\text { "using DERIVE" } \\
\text { Search in titles } \\
\text { (Google Scholar) }\end{array}$ & $\begin{array}{l}\text { \{With DERIVE\} } \\
\text { OR } \\
\text { \{using DERIVE }\} \\
\text { Search in titles } \\
\text { (Scopus) }\end{array}$ & $\begin{array}{l}\text { \{With DERIVE\} } \\
\text { OR } \\
\text { \{using DERIVE }\} \\
\text { Search in all fields } \\
\text { (Scopus) }\end{array}$ & $\begin{array}{l}\text { "with DERIVE" } \\
\text { OR } \\
\text { "using DERIVE" } \\
\text { Search in titles } \\
\text { (MathEduc) }\end{array}$ \\
\hline 1975-1979 & 0 & 0 & 0 & 0 \\
\hline 1980-1984 & 0 & 0 & 0 & 0 \\
\hline 1985-1989 & 1 & 0 & 0 & 0 \\
\hline 1990-1994 & 30 & 2 & 7 & 38 \\
\hline 1995-1999 & 56 & 2 & 11 & 64 \\
\hline $2000-2004$ & 27 & 0 & 9 & 41 \\
\hline 2005-2009 & 10 & 0 & 11 & 7 \\
\hline 2010-2014 & 5 & 0 & 26 & 2 \\
\hline 2015-Aug 2018 & 1 & 0 & 7 & 0 \\
\hline Total: & $136(130)$ & 4 & 71 & 152 \\
\hline
\end{tabular}

mentioning the use of DERIVE (third column of Table 4) appear well after the software is no longer commercialized.

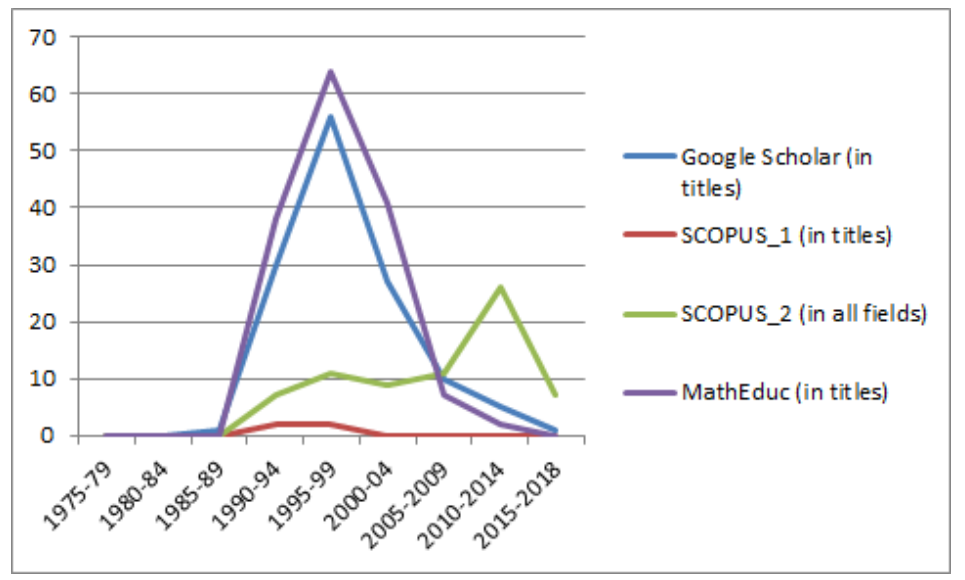

Fig. 7 Search for: with DERIVE $\vee$ using DERIVE in Google Scholar (in titles); in Scopus (1) (in titles); in Scopus (2) (in references) and in MathEdu (in titles). The graph corresponds to Table 4 .

5 So, is it only nostalgia or is there something special about DERIVE? (the authors' opinions)

Even if programming in DERIVE wasn't as simple or comfortable as in other CAS, still many math teachers that used it in the past consider it the sim- 
plest computer algebra system ever for beginners, and the most adequate for secondary/college education. This is probably because it was used "only" as a (powerful) algebraic calculator.

Some of DERIVE's advantages make sense no longer. For instance, the $M S$ $D O S$ version of DERIVE could run in PCs without a hard-disk (the initial versions were distributed in a single $5 \frac{1}{4}^{\prime \prime}$ diskette -later a $3 \frac{1}{2}^{\prime \prime}$ one) and the code of the last versions occupied only something like $2 M B$ of the CD. Nevertheless, other advantages, like its clear icons-oriented menus, are still remembered.

There were and are different approaches trying to simplify the use of computer algebra systems following DERIVE's approach. For instance, the early versions of wxMaxima, resembled DERIVE for Windows's front end, with a single input line in its lower part and output above in a $2 \mathrm{D}$ window (Figure 8). For instance [17] is an article about the possibility to substitute DERIVE with Maxima for educational purposes.

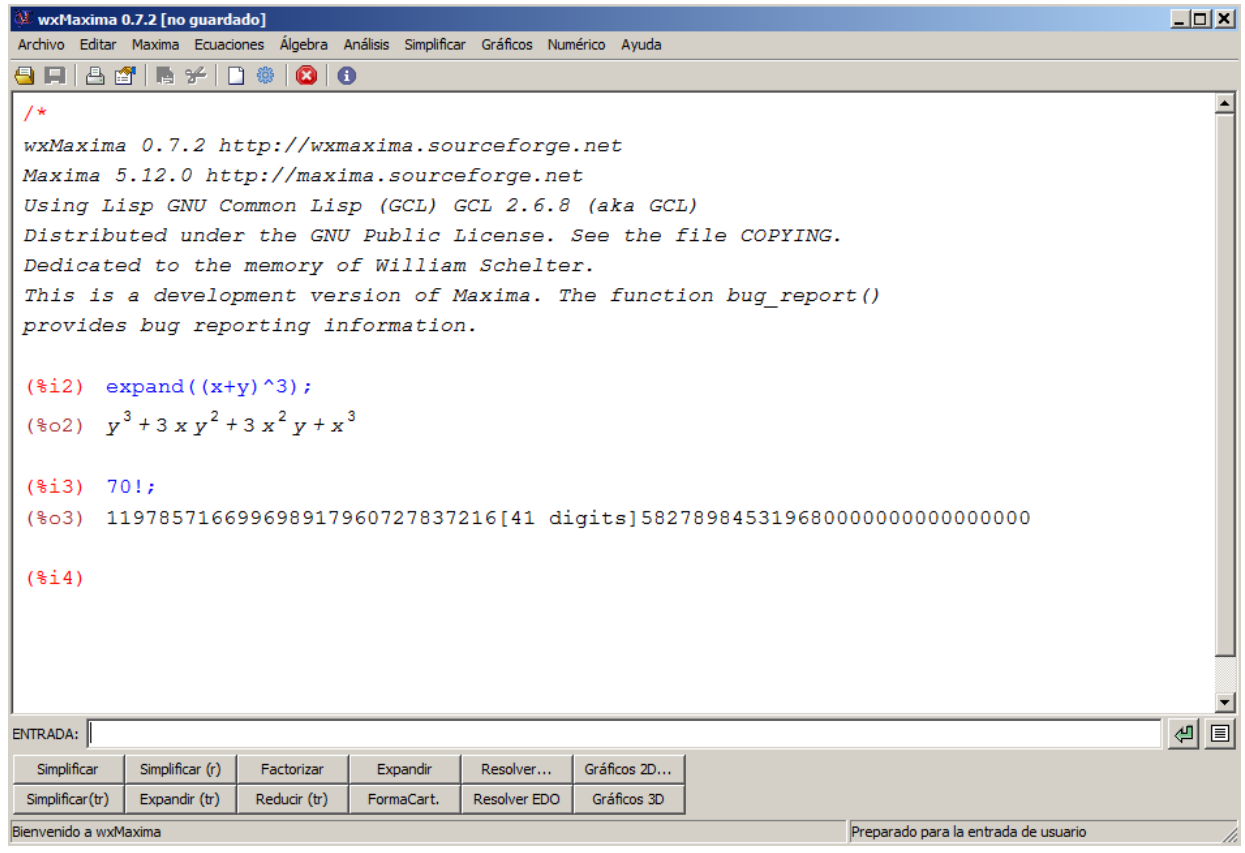

Fig. 8 The user interface of an early version (0.7.2) of wxMaxima.

Obviously other CAS follow other lines of development, like language flexibility: for instance, the astounding CAS Xcas allows four different syntaxes: its own, Maple's, MuPAD's and that of TI-89/92's calculators (it also incorporates a simple Dynamic Geometry System (DGS) plus Turtle Geometry). 
5.1 DERIVE's computational environment: pros and cons of the GUI and the programming capabilities

The GUI is very convenient, as it is oriented to dealing with buttons and menus, not with commands (although using commands and programming is also possible). Moreover, the number of buttons, menus and options is "appropriate" (there are enough for an average user and there aren't too many) and the "path" that has to be followed along buttons and menus is clear (it is not difficult even for a novice). Finally, the buttons have a "by default" option that is usually the desired one.

Note that it was mainly oriented as a symbolic calculator (the early versions only included the possibility to define functions -but not to define programs). An example of a user defined function is:

$F(n):=\operatorname{IF}\left(n<6, n^{\wedge} 2, n^{\wedge} 3\right)$

An example of procedure in the latter versions is:

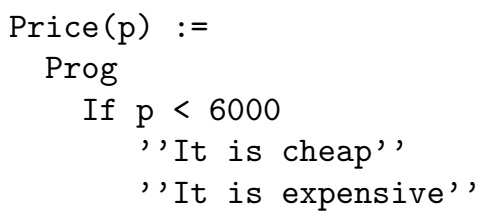

The editor for programs is poor. Moreover, a good program debugger is missing.

The usual loops: for, while, do while are not available (although it is possible to define other loops from DERIVE's LOOP command and there are two other related commands: ITERATE and ITERATES).

Many "built-in" commands and functions are available (and are complemented by user's libraries).

A disadvantage is the inflexible way to order new things to DERIVE (only available through the Input Line). It makes the Input/Output in the Algebra Window to have to be manually rearranged ("correctly ordered"). The flexibility of the usual 2-dimensional input/output windows of other CAS, where corrections can be introduced anywhere is missing.

We could also remark that DERIVE has an unfriendly editor for long lines of code.

5.2 DERIVE's computational environment: pros and cons of the computational environment (parser) and the plotting capabilities

The parser is surprisingly flexible and does an excellent work regarding patron recognition:

- the (by default) lack of distinction between uppercases and lowercases is good for novices, 
- the many different ways recognized to introduce functions: with or without parentheses, with or without spaces, allocating the exponents of functions in a flexible way (for example: $\sin ^{\wedge} 2 x, \operatorname{SIN}^{\wedge} 2 x, \operatorname{Sin}^{\wedge} 2 x, \sin ^{\wedge} 2 x, \ldots$ are all recognized as $\left.\sin (x)^{2}\right)$.

- the absence of statements separators or terminators (as a single entry line is used) is also convenient for newcomers.

The Algebra/2D/3D windows organization is flexible, allowing to easily copy plots into the worksheet.

Unfortunately, a plot command (2D and 3D) is clearly missing (plots can only be obtained using buttons or menus, and cannot be "ordered" from within a worksheet).

\subsection{DERIVE's computational environment: other pros and cons}

It was one of the pioneers in the step by step resolution (only available for certain commands). In user-developed programs, step by step resolution can be simulated using the DISPLAY command.

The two kind of files (MTH and DFW) allow to load complete worksheets and also to load "hidden code" (i.e., not echoed on the screen when loaded), although many other CASs offer this possibility too.

The I/O format is not standard and the program cannot be easily called from other applications (for instance just to perform a symbolic computation -in the way that, for example, Sage works).

Moreover, It was only available for MS-DOS/Windows platforms.

Finally, the number of available packages and options for the commands is small in comparison with the huge CAS like Maple or Mathematica (but consider that, for instance, the last distributions of these latter CAS come in a DVD).

\section{Conclusions}

Most surprisingly for a piece of software, the DERIVE community is still active (from the academic point of view) more than 10 years (!) after this CAS was discontinued.

In both the journals and conferences we can appreciate an evolution that has widened and updated the aims and scope although always around the initial DERIVE core.

Regarding the bibliographic data available, we could consider its evolution after it discontinuation as a decline slower than expected. Roughly speaking, the evolution of downloads and citations of a paper is considered to follow a negative exponential decay [30]. In this case, although the number of citations follow the pattern the decay is really slow (like a radioactive isotope with a long average life). 
One of the most appreciated characteristic of DERIVE was its very simple and flexible syntax (in opposite to most CAS which require a fixed syntax). Another important feature was the possibility of using not only the functions and programs in the main core of DERIVE or in its loadable packages, but also the multiple specific packages developed by users all over the world, increasing this way the capabilities of the CAS. For example, the two first authors of this paper have different packages published in the last release of DERIVE.

DERIVE possibly arrived in the right moment, as it was the only CAS that could be used on small computers (there was no competitor from this point of view). At the same time it was very friendly if used as an algebraic calculator (its most frequent purpose).

Something similar (arriving in the right moment) has happened in the XXIst century with the free DGS GeoGebra, with a surprising success and now almost monopolizing the DGS market. After incorporating CAS capabilities, it has been adopted as the CAS for educational purposes in many places.

After more than 10 years from the discontinuation of DERIVE, it has been used in the last years not only in education, but also in different areas of research such as music [3] or simulation of car traffic control [2].

As a final conclusion, we believe there is a market niche for a small CAS or a lite version of a CAS with an extremely short training period. So far no such a version of the big CAS exists.

Acknowledgements This work was partially supported by the research projects TIN201566471-P (Government of Spain) and CASI-CAM S2013/ICE-2845 (Comunidad Autónoma de Madrid).

\section{References}

1. Aguilera, G., Galán, J. L., Galán, M. A. and Rodríguez, P. Teaching semantic tableaux method for propositional classical logic with a CAS. The International Journal for Technology in Mathematics Education 22(2), 85-91 (2015).

2. Aguilera, G., Galán, J. L., García, J. M., Mérida, E. and Rodríguez, P. An acceleratedtime simulation of car traffic on a motorway using a CAS. Mathematics and Computers in Simulation 104, 21-30 (2014).

3. Aguilera, G., Galán, J. L., Madrid, R., Martínez, A. M., Padilla, Y. and Rodríguez, P. Automated generation of contrapuntal musical compositions using probabilistic logic in DERIVE. Mathematics and Computers in Simulation 80(6), 1200-1211 (2010).

4. Aguilera-Venegas, G., Galán-García, J. L., Galán-García, M. A., Lobillo-Mora, G., Martínez-del-Castillo, J., Merino-Córdoba, S., Padilla-Domínguez, Y., Rodríguez-Cielos, P. and Rodríguez-Cielos, R. Parametrization of curves and line integrals with a CAS. The International Journal for Technology in Mathematics Education 24(4), 179-190 (2017).

5. Anonymous. Macsyma, Mathematics and System Reference Manual. Macsyma, Inc., Arlington, MA (1996)

6. Aspetsberger, K. and Funk, G. Experiments with muMATH in Austrian high schools. ACM SIGSAM Bulletin 18-19(4-1), 4-7 (1984-85).

7. Barzel, B. (Ed.). Teaching Mathematics with Derive and the TI-92. Proceedings of the International Derive and TI-92 Conference, Schloss Birlinghoven. ZKL Texte Nr. 2. ZKL, Münster, Germany (1996)

8. Beaudin, M. Using $\ln (|x|)$ as an Antiderivative for $\frac{1}{x}$ is a Bad Choice!, in Wester, M. J. and Beaudin, M. (Eds.) Computer Algebra in Education. (p. 55-70). Aulonna Press, El Dorado, White Lake, MI, USA (2008). 
9. Beaudin, M., Henri, F. and Savard, G. Integration of Piecewise Continuous Functions. The Derive Newsletter 91, 321 (2013).

10. Böhm, J. (Ed.). Teaching Mathematics with Derive. Chartwell-Bratt, Bromley, Kent, UK. (1992),

11. Böhm, J. Dimensional analysis with DERIVE. Mathematics and Computers in Simulation 45(1-2), 197-205 (1998).

12. Burrel, F., Cabezas J. and Roanes-Lozano, E. and Roanes-Macías, E. A Survey on the Use of Computer Algebra in Spain in Relationship to Its Secondary School System. Zentralblatt für Didaktik der Mathematik 97(5), 149-154 (1997).

13. Cabezas, J. Una aplicación de DERIVE a la clase de Matemáticas. Boletín de la Sociedad "Puig Adam" de Profesores de Matemáticas 46, 71-78 (1997).

14. Cabezas, J. and Roanes-Lozano, E. Towards the Abandonment of Statistical Tables. Pro Dialog. Journal of the Polish Information Processing Society 12, 67-76 (2001).

15. Cabezas, J. and Roanes-Lozano, E. Four experiences and some reflections about the influence of mathematical software on the mathematics curriculum. Journal of Scientific Research and Reports 7(2), 154-164 (2015).

16. Galán-García, J. L., Aguilera-Venegas, G., Galán-García, M. A., Rodríguez-Cielos, P. and Atencia-Mc.Killop, I. Improving CAS capabilities: New rules for computing improper integrals. Applied Mathematics and Computation 316, 525-540 (2018).

17. García, A., García, F., Rodrguez-Sánchez, G. and de la Villa Cuenca, A. Could it be possible to replace DERIVE with MAXIMA? International Journal for Technology in Mathematics Education 18/3, 137-142 (2011).

18. Hearn, A. C. REDUCE User's Manual, Version 3.5. RAND Publication CP 78 (1993).

19. Hearn, A. C. REDUCE User's Manual, Version 3.8. Santa Monica, CA, USA (2004).

20. Hearn, A. C. REDUCE: The First Forty Years. (2005). Available from: http://www. reduce-algebra.com/reduce40.pdf

21. Heck, A. Grand Tour of Derive. CAN Expertise Center, Amsterdam, The Nederlands (1995). Available from: http://www.computeralgebra.nl/systemsoverview/general/ derive/grandtour/derive.html

22. van Hulzen, J. A. and Calmet, J. Computer algebra systems, in Buchberger, B., Collins, G. E. and Lees, R. (Eds.) Computer Algebra, Symbolic and Algebraic Manipulation. Springer-Verlag, Vienna (1983).

23. Jenks, R. D. and Sutor, R. AXIOM: the scientific computation system. Springer-Verlag New York, Inc., New York, NY, USA (1992).

24. Kutzler, B. Mathematics on the PC-Introduction to DERIVE. Soft Warehouse Europe, Hagenberg, Austria (1994).

25. Kutzler, B. Improving mathematics teaching with DERIVE. Chartwell-Bratt., Bromley, UK (1996).

26. Kutzler, B. Past and Future of Computer Algebra in Mathematics Education. (2010). Available from: http://www.time2010.uma.es/Proceedings/Papers/A045_Paper.pdf

27. Kutzler, B. and Kokol-Volc, V. Introduction to DERIVE 5. Texas Instruments (2000).

28. Kutzler, B. and Kokol-Volc, V. Introduction to DERIVE 6. Texas Instruments (2003).

29. MacCallum, M. A. H. and Wright, F. J. Algebraic Computing with REDUCE. Oxford University Press, Oxford, UK (1991).

30. Moed, H. F. Statistical relationships between downloads and citations at the level of individual documents within a single journal. Journal of the American Society for Information Science and Technology 56(10), 1088-1097 (2005). DOI: 10.1002/asi.20200

31. Moses, J. Macsyma: A personal history. Journal of Symbolic Computation 47(2), 123130 (2012). DOI: 10.1016/j.jsc.2010.08.018.

32. Pavelle, R. and Wang, P. S. MACSYMA from F to G. Journal of Symbolic Computation 1, 69-100 (1985).

33. Rayna, G. REDUCE Software for Algebraic Computation. Springer-Verlag, New York, Berlin (1987).

34. Rich, A. D. muLISP-87 LISP Language Programming Environment. Soft Warehouse, Inc., Honolulu, HI, USA (1987).

35. Rich, A. D., Rich, J. and Stoutemyer, D. R. Derive user manual. Version 2. A mathematical assistant for your personal computer. Soft Warehouse, Inc., Honolulu, HI, USA (1990). 
36. Rich, A. D., Rich, J. and Stoutemyer, D. R. Derive user manual. Version 3. A mathematical assistant for your personal computer. Soft Warehouse, Inc., Honolulu, HI, USA (1994).

37. Rich, A. D. and Stoutemeyer, D. R. Capabilities of the MUMATH-78 computer algebra system for the INTEL-8080 microprocessor, in EUROSAM '79 Proceedings of the International Symposium on Symbolic and Algebraic Computation. Springer-Verlag, London, UK (1979), 241-248.

38. Roanes-Lozano, E. An overview of the evolution of the devices running computer algebra systems and their educational implications. Electrical and Electronic Technology Open Access Journal 1(1), 7-11 (2017).

39. Roanes-Lozano, E., Cabezas, J., Vara, M. V., Roanes-Macías, E., Ortega, P. and Romo C. A Proposal for Filing the Gap Between the Knowledge of a CAS and its Application in the Classroom, in Wester, M. J. and Beaudin, M. (Eds.) Computer Algebra in Education. Aulonna Press, El Dorado, White Lake, MI, USA (2008), 7-17.

40. Schmidt, K. and Trenkler, G. Moderne Matrix-Algebra: mit Anwendungen in der Statistik. Springer-Verlag, Berlin (1998).

41. Texas Instruments TI-nspire CAS+. Math and Science Learning Technology Handheld. Texas Instrumens U.S.A., Dallas, TX (2017)

42. Wester, M. J. Computer Algebra Systems: A Practical Guide. John Wiley \& Sons., Chichester, UK (1999).

43. http://math.unm.edu/aca.html

44. http://www.acdca.ac.at/kongress/

45. http://axiom-developer.org/

46. http://www. austromath.at/dug/

47. http://www.freeoffice.com/

48. http://www.researchinformation.co.uk/time.php

49. http://www.issac-conference.org/

50. http://rfdz.ph-noe.ac.at/fileadmin/Mathematik_Uploads/ACDCA/Konferenzen.pdf

51. http://maxima. sourceforge.net/

52. https://www.openoffice.org/

53. https://education.ti.com/en/products/computer-software/ti-nspire-teacher-sw

54. https://reduce-algebra.sourceforge.io/

55. https://www. wordperfect.com/en/

56. http://www. wordstar.org/index.php/wordstar-history

57. https://en.wikipedia.org/wiki/WordStar

58. https://en.wikipedia.org/wiki/List_of_computer_algebra_systems

59. http://andrejv.github.io/wxmaxima/ 